\title{
L'Avenir de la Forêt Privée Québécoise" Tel que vu par la Section Orléans de l'Institut Forestier du Canada ${ }^{1}$
}

\section{Résumé}

Pour répondre à l'invitation lancée par le comité consultatif sur l'avenir de la forêt privée, la section Orléans a préparé un mémoire qu'elle a présenté le 16 février 1984. Le comité consultatif formé par le ministère de l'Energie et des Ressources ainsi que par la Fédération des producteurs de bois du Québec voulait savoir de la population:

1. comment elle voit l'aménagement des bois privés;

2. quel type de financement elle envisage pour la réalisation de cet aménagement;

3. si la législation existante entourant l'aménagement des bois privés devait être modifiée; et

4. quelle sorte de bois les propriétaires privés devaient produire en aménageant leur bois pour une mise en marché et une transformation efficace.

Le mémoire de la section Orléans a été très bien reçu par le comité consultatif. Voici les recommandations qu'il contenait:

1 Mémoire présenté devant le comité consultatif sur l'avenir de la forêt privée le 16 février 1984.
1. La forêt privée est importante et son aménagement devrait être intensifié.

2. Le rôle de la forêt privée devrait être défini régionalement compte tenu de son importance.

3. L'aménagement de la forêt privée devrait être orienté vers la production de bois-d'oeuvre dans la mesure du possible. A cet égard des actions, sur la fiscalité et la taxation municipale, devraient être mises de l'avant afin d'inciter le propriétaire à conserver son bois sur pied jusqu'à ce qu'il soit apte à faire du bois d'oeuvre de grande qualité.

4. L'éducation des propriétaires de bois devrait être accentuée.

5. Les OGC répondent à une certaine clientèle et devraient être maintenus; l'aide individuelle devrait être instaurée pour les autres.

6. L'encadrement technique devrait être mieux organisé et augmenté.

7. Le régime des dépenses gouvernementales en foresterie devrait être augmenté et orienté en fonction des objectifs de production régionaux.

8. Les responsables de l'application de l'entente MAPAQ-MER devraient agir avec plus de souplesse en ce qui concerne le reboisement de certaines terres.
9. L'importance relative des différents traitements devrait être établie en fonction des rendements désirés dans le temps.

10. La régénération naturelle des peuplements devrait être favorisée de préférence au reboisement dans la mesure du possible.

11. L'établissement d'une bonne régénération est essentiel, mais encore faudrait-il en assurer la survie et la croissance. A cet égard, les moyens d'y parvenir sont: les coupes de régénération et les préparations de terrain adéquates; le choix des essences et la mise en terre de plants vigoureux, ainsi que les dégagements et les éclaircies appropriées. Ces traitements devraient être faits en tenant compte des activités récréatives, de la production faunique, des sols, de l'eau, de la tordeuse, de la livrée, etc.

12. La fertilisation ne devrait être effectuée que dans les cas spéciaux comme, l'augmentation de valeur de peuplements de haute qualité et de vergers à graines.

13. Les travaux de drainage ne devraient être encouragés que dans les régions où la superficie à aménager est insuffisante.

Marcien Roberge, ing.f.

\section{The CIF/IFC on Parliament Hill}

The phone rang the other day and there was Frank Oberle on the line. Mr. Oberle is M.P. for Prince George Peace River and is Progressive Conservative Party critic on forestry matters. Frank was calling to let me know that the Standing Committee on Fisheries and Forestry would be focussing on forestry matters on May 15 and 29 and on June 12. The first two days will be on estimates of Government Canada, however, on the June 12 session the Committee wants to hear from outside agencies. His query was, "Can I book the Institute to appear?" My response was short and enthusiastic, "you bet". By the time you read this, your CIF representatives, President Paillé, Past President Cayford, industrial forester Bill Brown and your Executive Director will have appeared "for forestry". We shall report in the August issue of The Forestry Chronicle.

R.J. Bourchier

Executive Director 